\title{
Integration of Phase-Locked Loop Based Real-time Oscillation Tracking in Grid Synchronized Systems
}

\author{
Brad Trento, Bin Wang, Kai Sun, and Leon M. Tolbert \\ Department of Electrical Engineering and Computer Science \\ The University of Tennessee \\ Knoxville, TN USA \\ Emails: btrento@utk.edu, bwang13@utk.edu, kaisun@utk.edu, and tolbert@utk.edu
}

\begin{abstract}
This paper discusses how a real-time electromechanical oscillation monitoring system (REOMS) can be incorporated into synchrophasor devices. Using a closed loop feedback system consisting of a second order generalized integrator phase-locked loop (SOGI-PLL), this paper shows that real-time frequency, phase, and magnitude of electromechanical oscillations can be tracked using existing synchronized measurement platforms. The approach is demonstrated using simulation and a scaled hardware demonstration platform developed by the Center for Ultra-wide-area Resilient Electric Energy Transmission Networks (CURENT) to test wide-area monitoring and control schemes. Compared to traditional spectral base techniques, the phase lock loop approach offers more flexibility in tracking realtime oscillations, especially when the signal has floating modal properties. Real-time tracking of oscillation becomes extremely important when trying to adopt wide-area control schemes into the power grid, specifically oscillation damping and angular stability.
\end{abstract}

Index Terms-phase-locked loop (PLL), phasor measurement unit (PMU), real-time electromechanical oscillation monitoring system (REOMS), second order generalized integrator (SOGI), synchrophasor

\section{INTRODUCTION}

In the last decade, a large amount of research has been done on phase-locked loop (PLL) technology for grid monitoring and synchronization. The vast majority of the work has been in response to the growth of power electronics in utility applications; however, the use of PLL technologies in phasor measurement units (PMUs) and other frequency measurement applications has also received a great deal of attention in recent years. The goal of this work is to look at incorporating these techniques developed for grid synchronization into a real-time electromechanical oscillation monitoring system (REOMS). The work will begin by briefly discussing PLL technology but will mainly focus on a particular PLL implementation centered around the use of the second order generalized integrator (SOGI-PLL). There are many different techniques available for grid synchronization and it is not possible to evaluate every technique in this paper for use in real-time oscillation detection. However, the authors will discuss why some of the common techniques used for grid synchronization are not applicable for this application. The paper will discuss the details on how to design the controls for use in a REOMS system and then show how the frequency, angle, and magnitude can be tracked in real-time using both a software and hardware demonstration.
The authors in [1] first suggested using PLLs for oscillation detection because of the deficiencies in current spectral based techniques. One of the most notable advantages to come out of that paper was the ability to track, with high accuracy, electromechanical oscillations in real-time with a PLL. As the power industry moves towards measurement based widearea control systems, real-time detection methods are a must, and incorporating the functionality into the existing hardware is an attractive feature. This paper will expand upon that approach introduced by [1] using a more advanced PLL structure to provide better dynamic performance and accuracy along with describing how the control can be incorporated into the synchrophasor device to create a REOMS. The paper will also look at a marginally stable case to show how the frequency of the oscillation mode changes over time and how tracking is maintained with the REOMS.

In the rest of the paper, Section II briefly introduces the PLL technique and the proposed SOGI structure, Section III describes the details for designing the SOGI-PLL and incorporating it into existing synchrophasor devices, Section IV shows the simulation and experimental results of the proposed approach, and finally, conclusions are drawn in Section V.

\section{SOGI BASED PHASE-LOCKED LOOP}

A phase-locked loop is a type of closed-loop control system that automatically adjusts an internal oscillator to keep the time of some external periodic signal. The basic structure of the PLL is shown in Figure 1. It consists of the following three fundamental blocks [2]:

1) A phase detector (PD), used to generate an output signal proportional to the phase difference between the input signal and the signal generated by the internal oscillator of the PLL.

2) A loop filter (LF), used to attenuate the high frequency AC components of the PD. Typically, this block consists of a low pass filter, PI controller, or some combination of the two.

3) A voltage control oscillator ( $\mathrm{VCO}$ ), used to generate an AC signal whose frequency is shifted with respect to a center frequency as a function of the input voltage provided by the LF.

Since the PLL is a closed loop feedback system, the rebuilt signal can optimally fit the input singal, such that the frequency and phase can be dynamically estimated with high accuracy. 


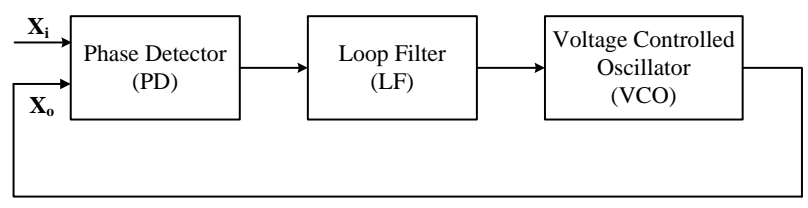

Fig. 1. Basic PLL structure.

The PLL technology used for grid synchronization is different than the traditional mixer type PLLs found in communication systems because of the grid operating requirements. This is mainly due to the transient and distortion requirements found in grid applications. Most communication type PLLs assume that the loop filter is tuned much lower than the source frequency so that the double frequency term after the PD is highly attenuated [2]. This type of PLL structure can result in a slow response when applied to grid synchronization systems because the loop filter needs to be tuned so low. One common workaround for single phase systems is to create a signal in quadrature with the input and then apply a dq transformation to transform the input signal to DC components. This allows the bandwidth of the controller to be increased while not sacrificing the accuracy of the measurement. In the remainder of the paper, the techniques developed for grid synchronization $(50-60 \mathrm{~Hz})$ will be used to track electromechanical oscillations $(\mathrm{mHz}-\mathrm{Hz})$. The next section will discusses the quadrature generator used in the PLL.

\section{A. SOGI-PLL}

In the last decade, there has been a great deal of work on how to obtain this quadrature signal from a single phase measurements for use in single phase PLLs. Some of the most notable methods include:
1) Differentiation [3]
2) Quarter cycle delay [4]
3) Hilbert transform [5]
4) Kalman filter [6]
5) Park/Inverse Park [7]
6) Adaptive filtering [8], [9], [10]

The SOGI method used in this paper is in the adaptive class of quadrature generators. Beyond the quadrature generator, most of the PLL structure is the same for phase locking techniques. The basic SOGI-PLL structure is shown in Figure 2. From the input signal, the SOGI quadrature generator creates an orthogonal signal $\left(\mathrm{X}_{\beta}\right)$ and also provides a filtered input signal $\left(\mathrm{X}_{\alpha}\right)$. The Park transformation $(\alpha \beta \rightarrow \mathrm{dq})$ applied after the SOGI-QG is defined in (1)

$$
T=\left[\begin{array}{cc}
\cos (\theta) & \sin (\theta) \\
-\sin (\theta) & \cos (\theta)
\end{array}\right]
$$

The basic structure of the SOGI quadrature generator is shown in Figure 3. The generation of the quadrature signal and filtered input signal is accomplished using two integrators in a feedback configuration. The transfer functions for filtered and orthogonal signal are given in (2) and (3). Figure 4 and 5 shows the bode plots of (2) and (3) for different values of

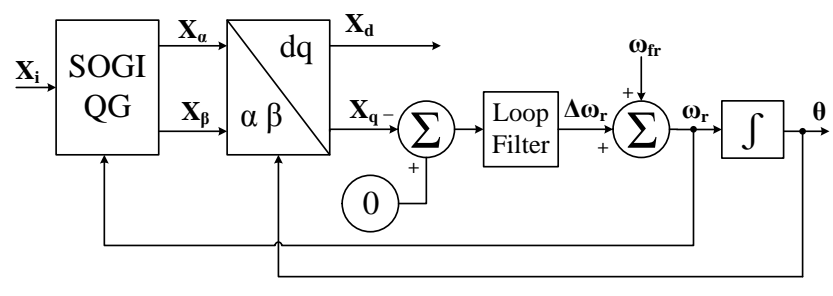

Fig. 2. Basic SOGI-PLL structure.

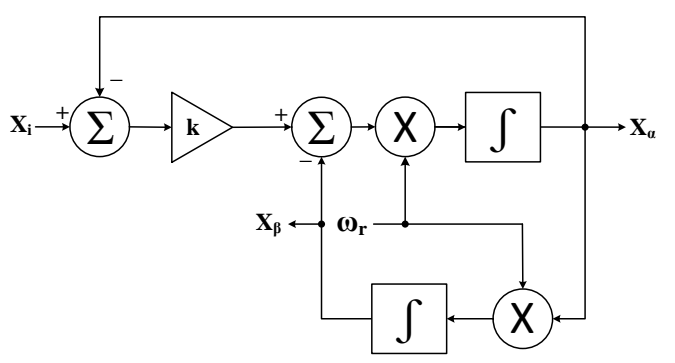

Fig. 3. SOGI quadrature generator.

$k$. For both figures, the resonant frequency is $2 \pi 0.5$. Based on the bode plots, the following can be concluded:

1) The transfer function $X_{\alpha}$ exhibits a bandpass filtering characteristic with a center frequency of $\omega_{r}$. The width of the passband is determined by the damping factor $\mathrm{k}$ and is independent of $\omega_{r}$. When the system frequency is equal to $\omega_{r}$, then $\mathrm{X}_{\alpha}$ will match in magnitude and phase with $\mathrm{X}_{i}$.

2) The transfer function $X_{\beta}$ exhibits a low-pass filtering characteristic. When the system frequency is equal to $\omega_{r}$, then $\mathrm{X}_{\beta}$ will be $-90^{\circ}$ out of phase with the fundamental component of the input signal $X_{i}$.

Lower $\mathrm{k}$ leads to better filtering capability. However, we can see from (2) and (3) that $\mathrm{k}<2$ leads to an underdamped response. Therefore, choosing the gain is not solely determined by the filtering aspects of the SOGI but a combination of filtering and dynamic performance.

$$
\begin{aligned}
& H_{\alpha}(s)=\frac{X_{\alpha}(s)}{X_{i}(s)}=\frac{k \omega_{r} s}{s^{2}+k \omega_{r} s+\omega_{r}^{2}} \\
& H_{\beta}(s)=\frac{X_{\beta}(s)}{X_{i}(s)}=\frac{k \omega_{r}^{2}}{s^{2}+k \omega_{r} s+\omega_{r}^{2}}
\end{aligned}
$$

\section{System INTEGRATION AND CONTROL DESIGN}

The complete system diagram of the REOMS including integration into the PMU is shown in Figure 6. For illustration purposes, the PMU implementation is shown with a synchronous reference frame PLL (SRF-PLL). This diagram is not implying though that the SRF-PLL is suitable for PMU applications. It is merely showing how the electromechanical oscillations can be detected from the grid synchronization PLL's frequency measurement. Most PLL based PMUs will have a similar structure where the nominal frequency $\omega_{f p}$ 


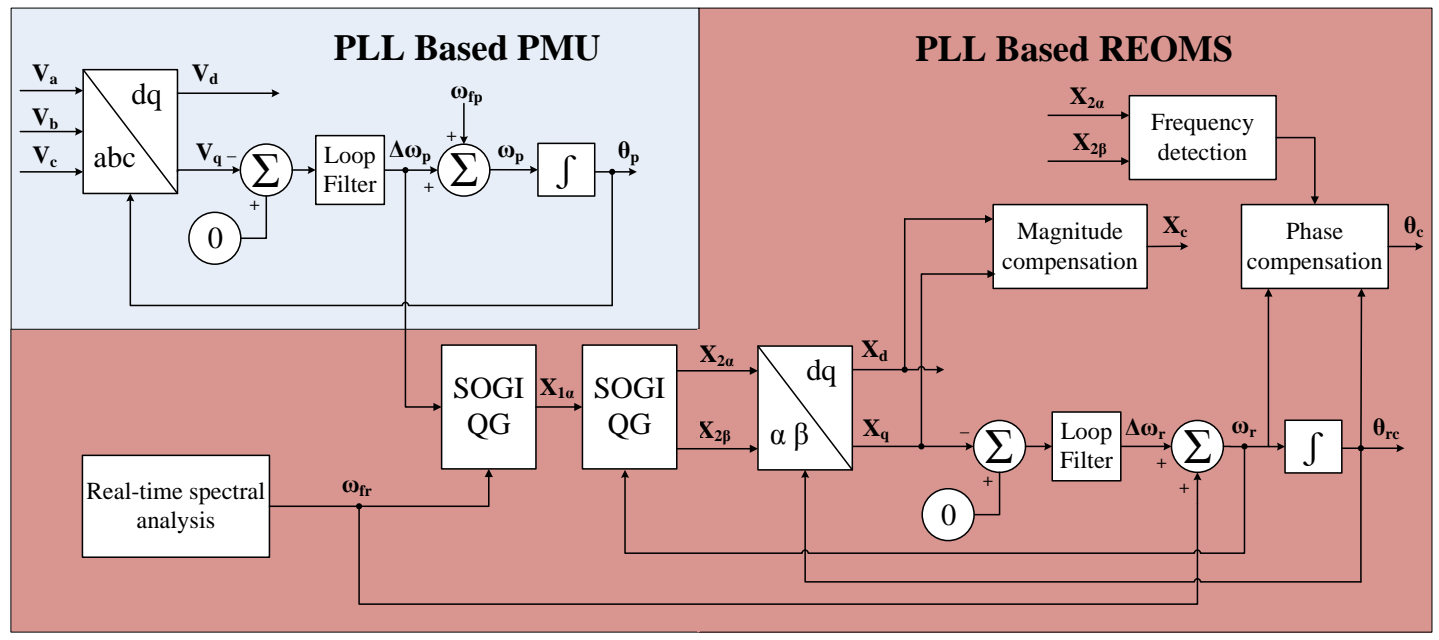

Fig. 6. REOMS integration in PMU.
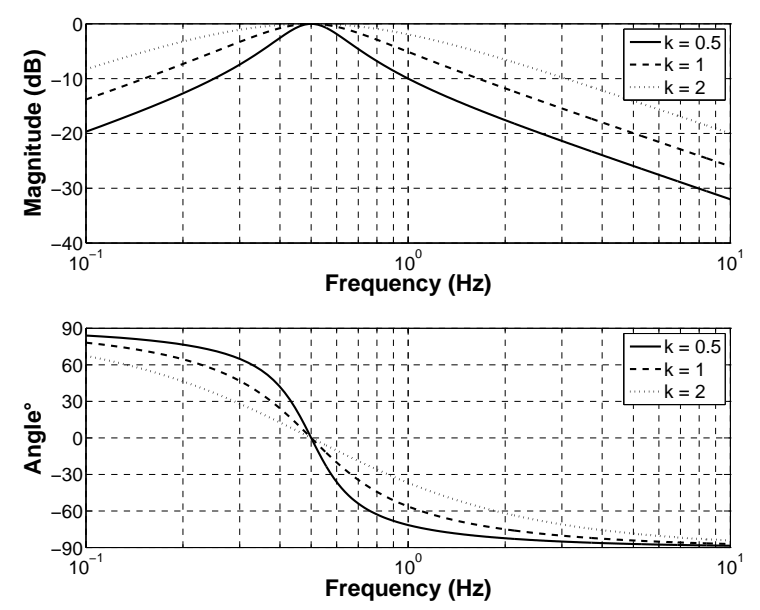

Fig. 4. Bode plot $\mathrm{v}_{\alpha} / v_{i}$.

is used to improve the start-up behavior of the PLL. The frequency error, $\Delta \omega p$, will be what is tracked by the REOMS. This frequency error will consist of a DC offset plus the oscillation frequency. The technique proposed in this paper can also be applied directly to the output of the PMU (i.e., the $30 \mathrm{~s} / \mathrm{sec}$ output). In fact, the continuous controls presented in the next section were discretized using a sampling rate of 30s/sec and Tustin's method to ensure proper operation when connected directly to the output of the PMU. If the REOMS is integrated into the PMU as shown in Figure 6, or with some other variation where the output signal is at a high sampling rate, the design is simpler and the numerical integration error discussed in [8] is negligible.

\section{A. DC Offset and Compensation}

Notice that the REOMS PLL shown in Figure 6 has two SOGI-QG, one being in feedback path and the other being from the real-time spectral block. The first SOGI is needed to remove the DC component from the $\Delta \omega_{p}$ measurement. Initially, the technique proposed in [11] was tried for this
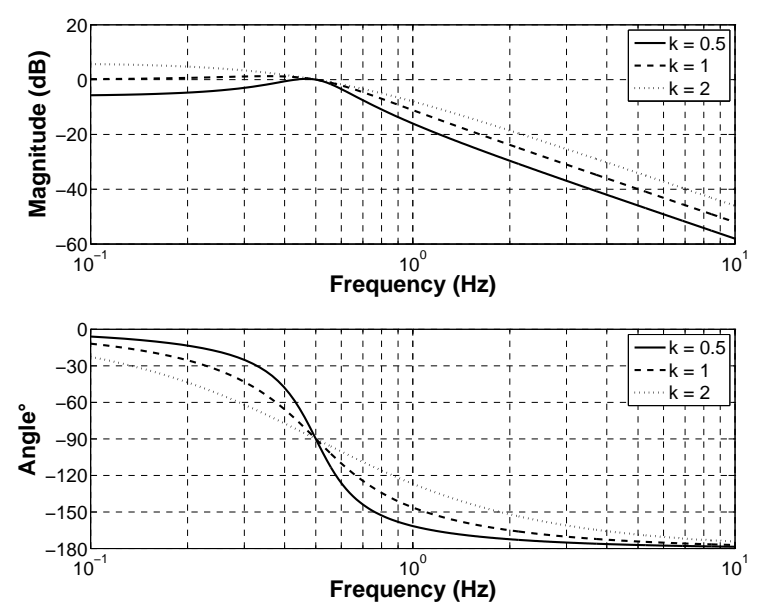

Fig. 5. Bode plot $\mathrm{v}_{\beta} / v_{i}$.

application, which would require only a single SOGI-QG, but when $X_{d c}>X_{a c}$ the system is unstable. Since the operating point is always changing in the power system, and the change can be quite significant following a disturbances, this method was not suitable for this application. This is also why some of the other common techniques like the Park-PLL and SOGIFLL cannot be directly applied in this application.

Since the transfer function for $X_{\alpha}$ has a zero at the origin, (2) can be applied to the input signal to remove the DC component. However, since it is not in the feedback path for this configuration, the phase and magnitude have to be compensated using the transfer function in (2). For example, the phase compensation is done using (4), where $\mathrm{k}$ is the damping coefficient discussed in the earlier section about the SOGI. The $\pi / 2$ in (4) is because $\theta_{r e}$ is bound between 0 and $2 \pi$ from the integrator used in the VCO. The magnitude after compensation, $X_{c}$, multiplied by $\cos \left(\theta_{c}\right)$ will be the 
reconstructed oscillation signal from the REOMS.

$$
\theta_{c}=\theta_{r e}+\operatorname{atan} 2\left(\frac{k \omega_{f r} \omega_{r}}{\omega_{f r}^{2}-\omega_{r}^{2}}\right)-\frac{\pi}{2}
$$

It is also possible to determine the phase and frequency through the frequency detection block. This block uses atan 2 of $X_{2 \alpha}$ and $X_{2 \beta}$, where atan2 is the atan of the two signal plus quadrant identification, and a derivative to get the frequency. The derivative is done using a difference equation with some additional logic to account for the changes from $\pi$ to $-\pi$ in the phase. The responses from the frequency detection block will be slightly quicker than the PLL output but is less immune to noise. The phase compensation (4) also needs to be applied to the phase angle for this approach.

\section{B. SOGI-PLL Controller Design}

A linearized model is presented for the SOGI-PLL in the following section. To derive the linearized model, the following assumptions are made about the system.

1) The frequency of the SOGI $\left(\omega_{r}\right)$ is close to the real frequency

2) There is only a small difference between the real and estimated phase angles

3) The input signal harmonics act as a disturbance to the feedback system

Starting from (2) and (3) and after applying Park's transformation (1), an approximate first order response can be derived for $\mathrm{v}_{d}$ and $\mathrm{v}_{q}$ for a step change in input. Since only $\mathrm{v}_{q}$ will be used in the control, the approximations are only given for the q-axis. The first order approximations are given in (5). The details about the derivation of (5) can be found in [12] and [2].

$$
\begin{gathered}
v_{q}(s) \approx \frac{V}{\frac{2}{k \omega} s+1} \phi_{e}(s)+D(s), \quad \text { when } k<2 . \\
v_{q}(s) \approx \frac{V}{\frac{k^{2}-1}{k \omega} s+1} \phi_{e}(s)+D(s), \quad \text { when } k \geq 2 .
\end{gathered}
$$

With the small signal model for the phase detector, the small signal model for the SOGI-PLL can be derived. Figure 7 shows the linearized model of the SOGI-PLL. The input signal and disturbances have been normalized where $\mathrm{D}^{\prime}(\mathrm{s})$ represents $\mathrm{D}(\mathrm{s}) / \mathrm{V}$. Assuming a PI controller as the loop filter, the openloop transfer function is given by (6).

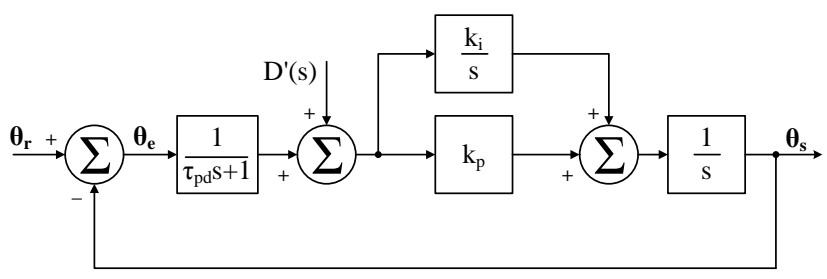

Fig. 7. Normalized linear model.

$$
\left.\frac{\theta_{s}}{\theta_{e}}\right|_{D^{\prime}(s)=0}=\frac{k_{i}\left(\tau_{f} s+1\right)}{s^{2}\left(\tau_{p d} s+1\right)}, \quad \text { where } \tau_{f}=k_{p} / k_{i} .
$$

From the small signal model, the controls were desiged using the technique of extended symmetrical optimum method discussed in [12].

\section{Simulation And Experimental Results}

The REOMS design was first tested using a known set of events in Simulink. Three events were applied to the REOMS, each with a different operating point followed by a ramp change in frequency from $0.4 \mathrm{~Hz}$ to $0.6 \mathrm{~Hz}$ at a rate of 0.01 $\mathrm{Hz} / \mathrm{s}$ and a damping of 0.2 . Figure 8 shows the frequency and magnitude results for this test. It is clear from the figure that the phase-locked loop in the REOMS does a good job of tracking the signal in real-time. The steady state error during the ramp is caused by a filter on the output of the frequency. Since the controller is type 2 (6), it can track a ramp with zero steady state error; however, some filtering is applied outside of the control loop that creates a small offset in the measurement.
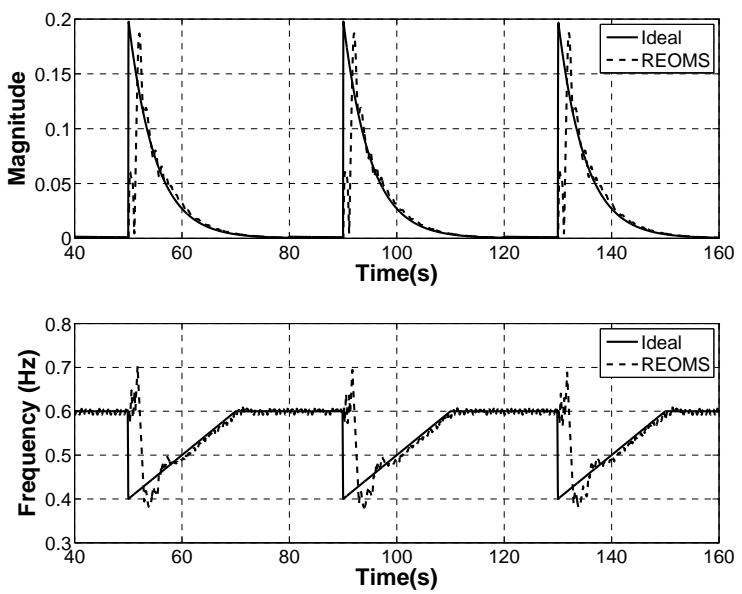

Fig. 8. Frequency ramp from $0.4 \mathrm{~Hz}$ to $0.6 \mathrm{~Hz}$.

The next test was done using a two generator system shown in Figure 9. For this set of tests, the clearing time of a temporary three phase fault at bus 1 was increased every $40 \mathrm{~s}$ to approach its critical clearing time to move the system closer to instability. The last event is a marginally stable case where the nonlinearity of the first swing is very pronounced. This test intends to show how the real-time oscillation frequency may fluctuate when the system behaves more nonlinearly. The top graph of Figure 10 shows the frequency difference between the two generators during the fault events vs. the reconstructed PLL output tracking this frequency difference. The bottom graph of of Figure 10 shows the real-time oscillation frequency of this frequency difference. By being able to track the oscillation frequency real-time, the nonlinearity of the event is captured giving more insight into the stability of the system. Spectral based techniques give an average of the event, so much of this nonlinearity is lost in the analysis.

The Center for Ultra-wide-area Resilient Electric Energy Transmission Networks (CURENT) has a hardware test bed with a scaled version of the two area system described in [13] emulated with power electronic converters with two SEL-421 PMUs and a few FDRs collecting synchronized measurement. 
Figure 11 shows the inter-area oscillation vs. the reconstructed waveform from the PLL from a test done in the HTB. It is clear from the figure that the PLL is able to track the oscillation in real-time very accurately. It should be noted that the transmission lines in the HTB emulation are discrete components, which is why the frequency of the mode is slightly different than what is presented in [13]. These discrete inductors are also one of the main reasons why the oscillations contains harmonics.

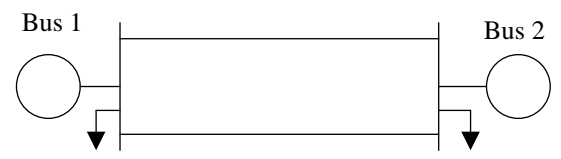

Fig. 9. Two generator system.
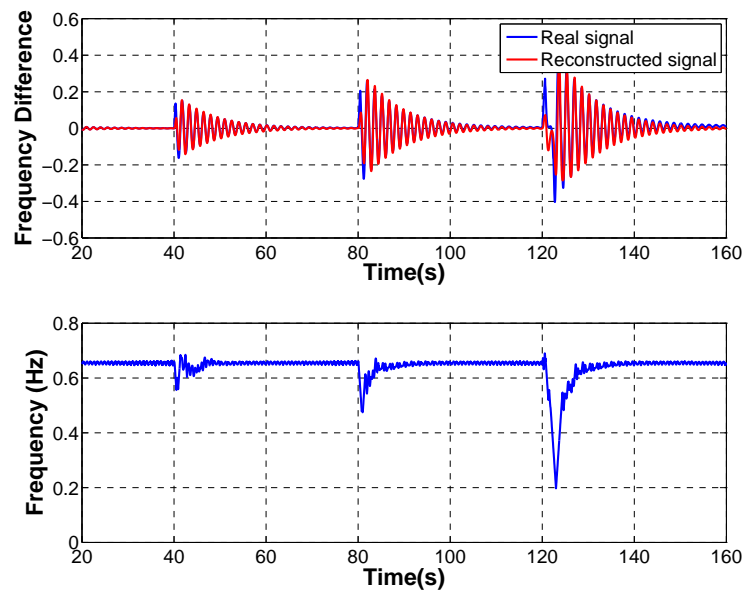

Fig. 10. Recontructed waveform (top) and real-time frequency of the event (bottom).

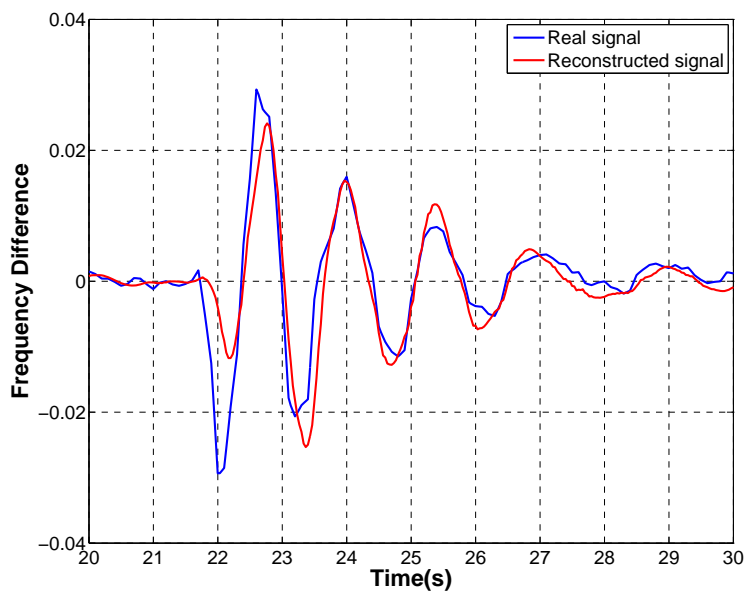

Fig. 11. Real vs. reconstruced waveform.

\section{CONCLUSION}

This paper discussed how real-time electromechanical oscillation monitoring systems (REOMS) can be incorporated into synchrophasor devices. Using a closed loop feedback system consisting of a second order generalized integrator phase-locked loop (SOGI-PLL), this paper showed that realtime frequency, phase, and magnitude of electromechanical oscillations can be tracked using existing synchronized measurement platforms. The approach was demonstrated using simulation and a scaled hardware demonstration platform. Compared to traditional spectral base techniques, the phase lock loop approach offers more flexibility in tracking realtime oscillations, especially when the signal has floating modal properties.

\section{ACKNOWLEDGMENT}

This work was supported primarily by the Engineering Research Center Program of the National Science Foundation and the Department of Energy under NSF Award Number EEC1041877 and the CURENT Industry Partnership Program.

\section{REFERENCES}

[1] K. Sun, Q. Zhou, and Y. Liu, "A phase-locked loop-based approach to real-time modal analysis on synchrophasor measurements," IEEE Trans. Smart Grid, in press.

[2] R. Teodorescu, M. Liserre, and P. Rodriguez, Grid Converter for Photovoltaic and Wind Power Systems. Hoboken, NJ: Wiley, 2011.

[3] A. Roshan et al., "A d-q frame controller for a full-bridge single phase inverter used in small distributed power generation systems," in Proc. IEEE 22nd Annu. Appl. Power Electron. Conf. (APEC), Feb. 2007, pp. 641-647.

[4] R. Zhang et al., "A grid simulator with control of single-phase power converters in d-q rotating frame," in Proc. IEEE 33rd Annu. Power Electron. Specialists Conf. (PESC), 2002, pp. 1431-1436.

[5] M. Saitou and T. Shimizu, "Generalized theory of instantaneous active and reactive powers in single-phase circuits based on hilbert transform," in Proc. IEEE 33rd Annu. Power Electron. Specialists Conf. (PESC), 2002, pp. 1419-1424.

[6] K. De Brabandere et al., "Design and operation of a phase-locked loop with kalman estimator-based filter for single-phase applications," in Proc. IEEE 32nd Annu. Industrial Electron. Conf. (IECON), Nov. 2006, pp. 525-530.

[7] L. Arruda, S. Silva, and B. J. C. Filho, "Pll structures for utility connected systems," in Proc. IEEE 36th Ind. Applicat. Conf. (IAC), Oct. 2001, pp. 2655-2660.

[8] M. Ciobotaru, R. Teodorescu, and F. Blaabjerg, "A new single-phase pll structure based on second order generalized integrator," in Proc. IEEE 37th Power Electron. Specialists Conf. (PESC), Jun. 2006, pp. 1-6.

[9] P. Rodriguez et al., "Grid synchronization of power converters using multiple second order generalized integrators," in Proc. IEEE 34th Annu. Industrial Electron. Conf. (IECON), Nov. 2008, pp. 755-760.

[10] M. Mojiri, M. Karimi-Ghartemani, and A. Bakhshai, "Estimation of power system frequency using an adaptive notch filter," IEEE Trans Instrum. and Meas., vol. 56, no. 6, pp. 2470-2477, 2007.

[11] M. Ciobotaru, R. Teodorescu, and V. Agelidis, "Offset rejection for pll based synchronization in grid-connected converters," in Proc. IEEE 23rd Annu. Appl. Power Electron. Conf. (APEC), Feb. 2008, pp. 1611-1617.

[12] S. Golestan et al., "Dynamics assessment of advanced single-phase pll structures," IEEE Trans. Ind. Electron., vol. 60, no. 6, pp. 2167-2177, 2013.

[13] M. Klein, G. Rogers, and P. Kundur, "A fundamental study of inter-area oscillations in power systems," IEEE Trans. Power Syst., vol. 6, no. 3, pp. 914-921, 1991.

[14] K. Sun et al., "An adaptive power system equivalent for real-time estimation of stability margin using phase-plane trajectories," IEEE Trans. Power Syst., vol. 26, pp. 915-923, 2001. 